\title{
Cellular distribution framework for wireless sensor based decision support systems for crisis management model
}

Sanjeev Gupta and Mayank Dave

Doeacc Society, Autonomous body of Department of Technology, Kurukshetra, India

Department of Computer Engg., National Institute of Technology, Kurukshetra, India

sanju_anita@yahoo.com; mdave@nitkkr.ac.in

\begin{abstract}
Crises management is a challenging problem for homeland security. The challenging aspect in crisis management is the early assessment of needs and damages. The existing approaches are quite unstructured in nature which results in poor resource management and hence inefficient. In this paper, the architecture for reliable and real time approach by using sensor clusters has been proposed for storage management. Instead of storing information in an individual cluster head as suggested in some approaches, storing of information of all clusters, inside the cell is recommended within the corresponding base station. It is assumed that the sensor nodes are aware of their locations in their deployment area, and they are time synchronized. For data dissemination and action in the wireless sensor network the usage of Action and Relay Stations (ARS) has been proposed. In the designed model sensor nodes are deployed prior to a crisis and the sensed information is stored. In case of emergency stored information is queried to get the report of humans trapped under rubble. It is also used to set the objectives and policies for emergency assistance.
\end{abstract}

Keywords: Crisis management; Data persistent; Decentralization; Reliability; Clustering; Base station; Action and Relay Stations (ARS).

Introduction

Crises management is a challenging problem for homeland security. Typically, crises management encompasses four phases of operation: 1) immediate response to the crisis for protecting lives and property, 2) recovery efforts in the aftermath of the crisis, 3) mitigation to lessen the impact of the crises, and 4) preparedness to learn from the outcome for future crises.

Responding to a disaster or a crisis in a timely and effective manner can reduce deaths and injuries, contain secondary effects, and reduce the resulting economic losses and social disruption. The reliable early warning system based on WSN can be deployed in areas with high risk of disasters. The use of WSN promise to provide real time information of the disaster area to rescue teams making coordination and planning more effective. Location information of victims, rescuers and objects in the disaster is vital for the rescue operations.

Sensor nodes can be used for continuous sensing, event detection, event identification, location sensing, and local control of actuators. Sensor nodes (Akyildiz et al, 2001; Jone \& Sivalingam et al., 2001) are expected to operate autonomously in unattended environments and potentially in large numbers. Failures are inevitable in wireless sensor networks due to inhospitable environment and unattended deployment.

Disasters are absolutely unavoidable, though a great amount of efforts have been made in the domain of crisis management and emergence response. The use of wireless sensor networks in emergency and disaster relief operations promise to provide real time information of the disaster area to rescue teams making coordination and planning more effective. The information of victims, rescuers and objects in the disaster is vital for the rescue operations. The information will absolutely help managers (Chen et al., 2006) make better decisions and take actions in time.

Crisis management must include combinations of analysis of accumulated data, i.e. detecting events, spatial information and distributed automated decision making and subsequent action on the basis of it. The rest of the paper covers: the requirement for effective disaster management, the design issues, description of the system architecture and the benefits of the proposed architecture.

Requirements for crisis management

Crisis management is a very complex process. In order to facilitate the meaningful design of wireless sensor networks for crisis management there are a number of requirements that needs to be fulfilled.

In all kinds of crisis, decision-makers will need to start by building up a picture consisting of the location of people, their conditions, their needs, the services still available.

\section{Physical attributes of sensors}

It is likely that the sensor nodes would be deployed in advance in the monitored area.

\section{Configuration}

Sensor nodes must be able to rapidly identify neighbors within communications range. They should be configured into an organized network without manual intervention. Moreover the formed network should be able to cope with node failures by implicit reconfiguration.

\section{Network size}

For the majority of operations the area to be covered by the network may be between 1-2 kms. Generally a communications range between nodes of around 250-500 $\mathrm{m}$ would be acceptable.

Collaborative objective

Typically, the objective of a sensor network is monitoring a certain signal of interest and informing a managerial entity about the activity in the region that is being sensed. Since a sensor network is deployed for
Research article

CIndian Society for Education and Environment (iSee)
"Crisis management using WSN" http://www.indjst.org
Gupta \& Dave Indian J.Sci.Technol. 
achieving a certain system-wide goal, nodes collaborate instead of competing with each other.

Redundancy

The system must guarantee serviceability in emergency situations. Due to the frequent node failures and inaccessibility of failed nodes, WSNs are required to have high redundancy so that effect of failure of few nodes becomes negligible and the objective should be achieved successfully.

\section{Data centric processing}

In WSNs sensor nodes are not accessed by a unique ID. Instead of addressing nodes with ID, it is more natural to access the data directly through content, attribute, e.g., location of node. For example, query will be to "collect readings in the region bound by the rectangle $(x 1, y 1, x 2$, $y 2)$ ", instead of "collect readings from a set of nodes of having addresses $\mathrm{x}, \mathrm{y}$ and $\mathrm{z}$."

\section{Data storage}

The collected data must be stored (Ganesan \& Greenstein et al., 2003), at least temporarily, within the network so that it can be queried dynamically by rescuers to effectively plan the rescue mission as well as to avoid dangers and also to know the potential cause of the disaster.

The queried data could be real-time as well as longterm data about the monitored area The stored data will also be required for analysis purpose in order to determine loopholes so that operational capability can be enhanced for preventing such kind of disasters in future.

\section{Data reliability}

In the sensor networks for disaster management data integrity and reliability are extremely important for taking effective decision. The reliability of data is crucial to take an effective decision.

Design issues

The sensor data behaves differently in many ways from the data in traditional databases

(Madden et al., 2003; Yao \& Gehrke 2002). The storage management problem can be dissected into following components:

- System support for storage management.

- Collaborative storage.

- Indexing retrieval.

and

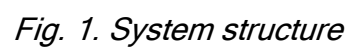

Fig. 1. System structure
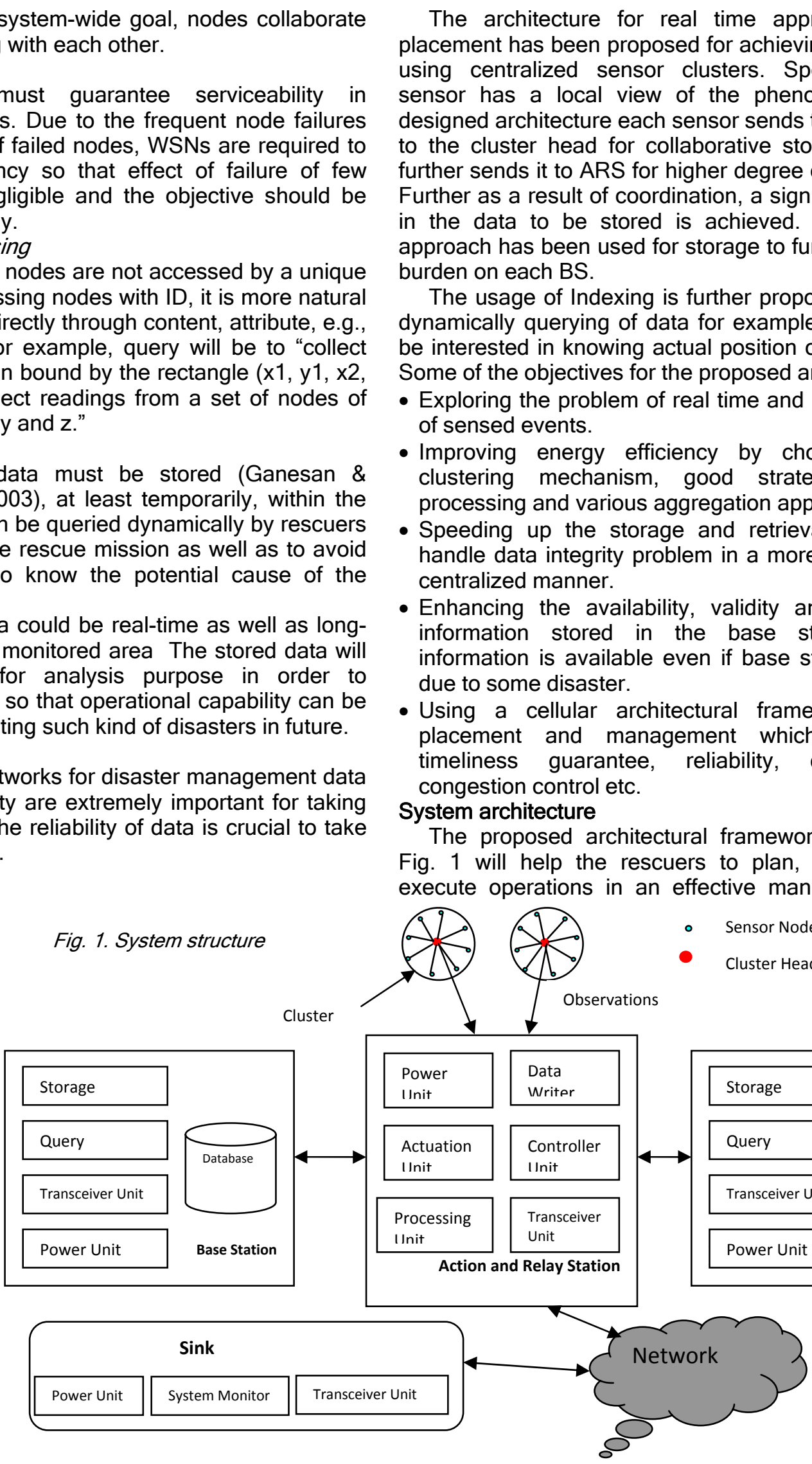

Research article

CIndian Society for Education and Environment (iSee)

"Crisis management using WSN" http://www.indjst.org
ISSN: 0974- 6846
Observations

The architecture for real time approach for data Specifically, each designed architcture vech sensor sends the sensed data to the cluster head for collaborative storage. Each $\mathrm{CH}$ further sends it to ARS for higher degree of collaboration. age to further reduce the den on each BS. . of sensed events.

by choosing suitable strategy for query - Speeding up the storage and retrieval of data and handle data integrity problem in a more controlled and station so that ter. reliability, data integrity,

\section{stem architecture}

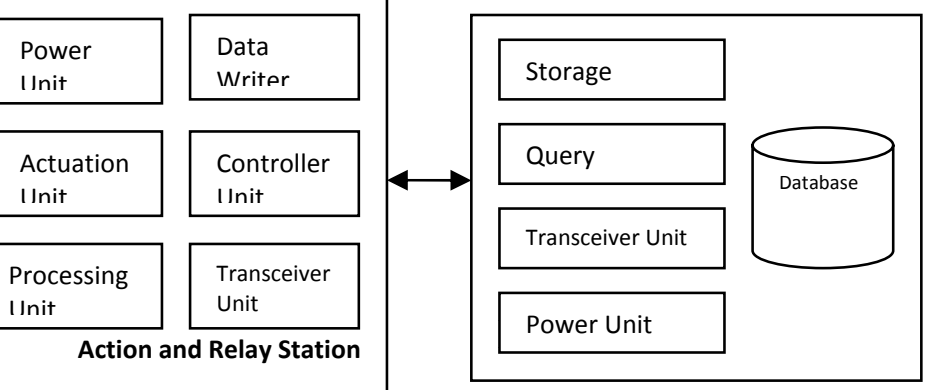

Action and Relay Station

Gupta \& Dave Indian J.Sci.Technol. 
fulfill the required mission objectives. The proposed model is based on distributed hierarchical framework. The proposed framework is portioned into components

Fig. 2. State transition at each sensor node

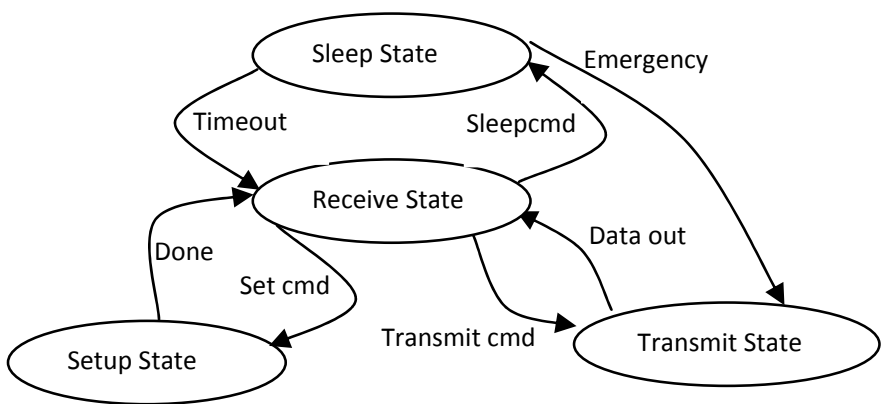

each of which will deal with various responsibilities of the overall system. The basic components and their functionalities are described briefly in following subsections.

Sensor nodes

Fig. 3. Cluster based sensor network

Sensors nodes are low-cost, low-power devices with limited sensing, computation and wireless communication capabilities. They can sense events in a circular coverage area with radius $r_{\mathrm{s}}$. To save energy as shown in Fig. 2 some sensors can be in sleeping state but they can be activated when it is necessary. Cluster structure

One of crucial challenges in the organization of sensor networks is energy efficiency, because battery capacities of sensor nodes are severely limited and replacing the batteries is not practical. Clustering (Yao \& Gehrke, 2002) is a standard approach for achieving efficient and scalable control in these networks.

Clustering saves energy and reduces network contention by enabling locality of communication: nodes communicate their data over shorter distances to their respective cluster-heads. Not all nodes, but only the cluster-heads need to communicate far distances to their respective action and relay station ARS (discussed in subsection 4.5).

The operation of cluster based sensor network is divided into rounds. Each round has two phases, the clustering phase and the data transmission phase. Rounds are repeated to monitor events continuously.

Research article

CIndian Society for Education and Environment (iSee)
As shown in Fig.3, the group of sensor nodes forms a cluster. To prevent draining of battery of a single sensor the cluster-head position is also randomly rotated among them. For cluster formation centralized clustering method (Heinzelman et al., 2000; Hill et al., 2000; Heinzelman et al., 2002) is used.

The centralized clustering will be better than the self organized clustering in terms of energy efficiency and prolonging network life time. In the clustering process a lot of energy is consumed during cluster formation due to communication among various nodes and also because clustering is a cyclic process.

Therefore instead of relatively low energy sensor nodes deciding among themselves the new leader in many rounds of communication and spending precious energy, it is better that a single energy and resource rich single entity takes a decision regarding the new leader. All the member nodes of the cluster will submit their candidature to this central entity.

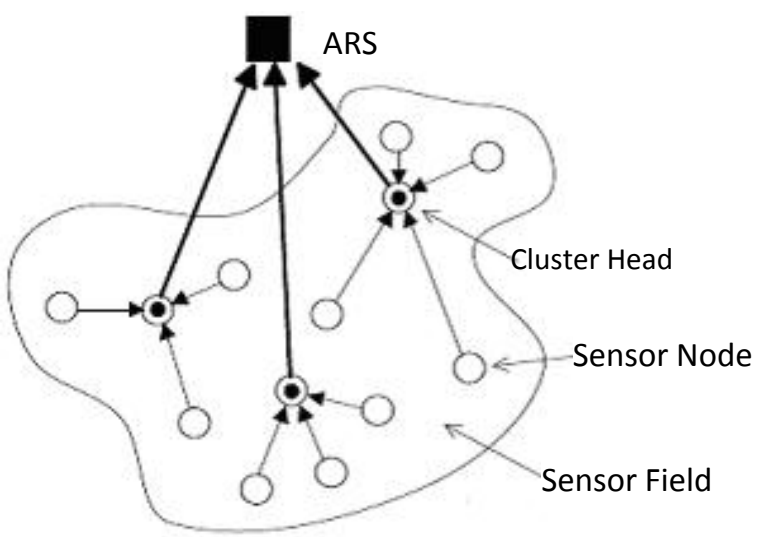

In the proposed model the ARS is the entity which will manage the clusters. Instead of all the sensor nodes of various clusters communicating to a single entity for decisions regarding the clustering, in the proposed architecture each ARS will only manage clusters belonging to it. Consequently in the proposed architecture clustering process will be speedier and energy efficient.

\section{Cell structure}

As shown in Fig.4 within every cell there will be number of clusters. An equal-sized cluster is a desirable property because it enables an even distribution of
Fig. 4. Various components of a cell

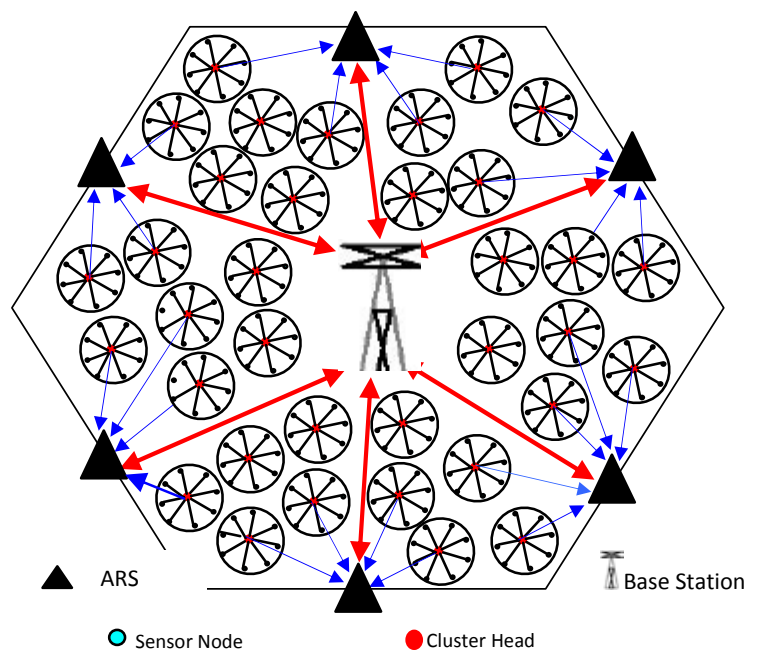

"Crisis management using WSN" http://www.indjst.org control (e.g., data processing, aggregation, storage load) over cluster-heads; no clusterhead is over-burdened or under-utilized. Minimum overlap among clusters is desirable for energy efficiency because a node that participates in multiple clusters consumes more energy by having to transmit to multiple cluster-heads.

Base station

The primary concern is the persistence of data, thus the goal is to minimize the amount of data lost due to failures of nodes as the emergency progresses (e.g., the fire 
spreads, rocks continue to bombard an area, or flood waters continue to grow). In wireless sensor network, the individual identity of nodes is always unimportant. For storing information, as suggested in (Heidemann et al., 2001; Ganesan et al., 2003; Furuta et al., 2006) the data centric storage has been proposed. In data centric storage, data can be stored and retrieved by name. It also enables use of indices or keys for efficient access to data in large-scale sensor networks. Further to speed up the storage and retrieval of data hashing mechanism can be used. The base stations are responsible for data storage in a distributed real time database framework.

The ARS lying on each pair of shared edges along the border between two cells passes the observations received from various $\mathrm{CHs}$ to the base stations of both the cells. In case base station receives redundant information it is ignored. The advantage of replication is that in case of failure of base station due to some disaster, ARS can obtain information from the adjacent cell's base station so as to take effective and reliable action. The aim is to prevent collapse of the system.

Action and relay station (ARS)

The ARS are resource rich nodes equipped with better processing capabilities, higher transmission powers and longer battery life. The ARS nodes are placed on the bordering areas of cells and are responsible for data dissemination in a time efficient manner.

During disaster any ARS may be damaged. But probability of collapse of all ARS's of the cell is very small. Only one ARS is enough to convey data from sensor network of a cell to a base station.

Sink

The sink acts as a bridge between wireless sensor network and physical world. The sink supervises and synchronizes the working of various components of WSN. It reports critical findings so that in case of some disaster or any other eventuality help from outside can reach the reported event area quickly. It monitors entire wireless network and depending upon feedback it sets the value of various controlling parameters like retention period so that the network can work efficiently.

Benefits of the proposed architecture

For an operationally effective disaster management, sensing, monitoring and decision-making should be integrated seamlessly. The proposed architecture is designed to meet these objectives. Some of the benefits of the proposed architecture which are critical for success of any disaster management operation are listed below.

\section{Actuation}

Much work has been focused on data collection, sensing and monitoring of the disasters targeted and energy consumption little research has been done on the
Vol.2 No. 11 (Nov. 2009)

ISSN: 0974- 6846 process of automatic decision making and subsequent actions during disasters. The sudden and timely actions will help in saving of invaluable human lives.

The ARS will dramatically extend the capabilities of a network in two ways: 1 ) it will enhance the sensing task, by pointing cameras, aiming antennae or repositioning sensors, and 2) it will retrieve as well as process the sensor data and performing appropriate actions automatically on the basis of it.

\section{Enhanced longevity}

The decentralized model for clustering and data storage will be more energy efficient than other centralized clustering approaches because average distance between cluster member nodes and respective ARSs will be much shorter than average distance between sensor nodes of clusters and the single base station of centralized clustering approaches. It will result in enhanced lifetime of the network.

\section{Data replication}

In the proposed architecture the information will be replicated and stored in a distributed manner, so that if any base station collapses, the required information could be retrieved from other base station. The distributed architecture also results in enhanced availability and speedier query processing.

\section{Cellular framework}

A new cellular architectural framework for reliable and real time data placement and dissemination of data in wireless sensor networks will provide timeliness guarantee, reliability, data integrity and congestion control.

\section{Data integrity}

Instead of storing information in individual $\mathrm{CH}$ as suggested in some protocols, in the proposed architecture, the storage of information of all clusters within a cell is suggested inside the corresponding base station. Therefore in the architecture data integrity problem has been handled in more controlled and centralized manner thereby resulting in accurate data for effective action.

\section{Faster data retrieval}

Using data centric storage mechanism in the architecture cost of accessing events (aggregation of observations generated by various sensor nodes) will be almost zero since all events will be available at one node (base station).

Results

For even moderately-sized networks with tens of nodes, it is extremely difficult to analytically model the interactions between all the nodes. Therefore to show effectiveness of the proposed model the simulation software has been developed to conduct the said
Research article

CIndian Society for Education and Environment (iSee)
"Crisis management using WSN" http://www.indjst.org
Gupta \& Dave Indian J.Sci.Technol. 
experiments. In the simulation 96 sensor nodes were randomly distributed in a cellular region of $120 \mathrm{~m} \times 120 \mathrm{~m}$.

To simplify the analysis we have assumed maximum of 24 clusters and maximum of 4 clusters per ARS. The data transmissions from sensor nodes were simulated until all sensor nodes died. For the experiments described here the parameters as described in Table 1 were used. For energy dissipation the model explained in (Heinzelman et al., 2002) have been used, that depends on the distance between the transmitter and receiver.

The performance of proposed model has been compared with popular Centralized-Clustering algorithm Leach-C taking exactly same environment and same assumptions.

Although Leach-C appears to be promising centralized clustering algorithm, there are some areas for making protocol more energy efficient. Instead of all the sensor nodes of the cell communicating to single BS, the proposed distributed model will be more energy efficient because average distance between cluster member nodes and respective ARSs will be much shorter than average distance between sensor nodes of clusters and the single base station of centralized clustering approaches.

Moreover instead of communicating and checking battery level of all the nodes of the cell to decide clustering, the ARS will only communicate with nodes reporting to it. Thus clustering process will be completed much faster and results in minimum use of invaluable energy of nodes and ARS.

The number of rounds that first node and last node dies as well as average energy dissipation is used as a key indicator to evaluate the proposed system. Experiment shows that our proposed model increases the lifetime of the network as illustrated in Fig.5. The scalability is also very easy to achieve in our model.

Conclusion

The distributed clustering method has been proposed to increase the longevity of the network. The proposed model not only offers real time sensing and monitoring, but also provides automated action in case of crisis. The model is easily scalable. It improves coordination among various entities and provides accurate as well as integrated information.

A lot more power-efficient designs can be produced from this framework. Similarly, there is also a need for synchronization among different sensors reporting the same event to multiple or same ARS. The architecture assumes that nodes know their physical positions, which is not true for many applications.

\section{References}

1. Akyildiz IF, Su W, Sankarasubramaniam $Y$ and Cayirci E (2002) Wireless Sensor Networks: A survey. Computer Networks. 38(4), 393-422.

2. Chen LC, Liu YC and Chan K-C (2006) Integrated communitybased disaster management program in Taiwan: a case study of Shangan village. Natural Hazards. 37(1-2), 209-

\section{3.}

3. Furuta T, Sasaki M, Ishizaki F, Suzuki A and Miyazawa $H$ (2006) A new cluster formation method for sensor networks using facility location theory. Nanzan Univ., Technical Report 01. http://www.seto.nanzanu.ac.jp/msie/nas/techreport/ index.html.

4. Ganesan D, Estrin D and Heidemann J (2003) DIMENSIONS: Why do we need a new data handling architecture for sensor networks? ACM SIGCOMM Computer Commun. Rev., ACM, NY, USA. 33(1), 143148.

5. Ganesan D, Greenstein B, Perelyubskiy D, Estrin D, and Heidemann J (2003) An evaluation of multi-resolution storage for sensor networks. Proc. $1^{\text {st }}$ Intl. Conf. on Embedded Networked Sensor Sys. ACM Press. pp: 89102.

6. Heidemann J, Silva F, Intanagonwiwat C, Govindan R, Estrin D and Ganesan D (2001) Building efficient wireless sensor networks with low-level naming. Proc. of the 18th ACM Sym. on Operating Sys. Principles, Banff, Alberta, Canada. pp: 146-159.

7. Heinzelman WB, Chandrakasan AP and Balakrishnan $\mathrm{H}$ (2002) An application-specific protocol architecture for wireless microsensor networks. IEEE Trans. on Wireless Commun. 1(4), 660- 670.

8. Heinzelman WR, Chandrakasan A and Balakrishnan $\mathrm{H}$ (2000) Energy-efficient communication protocol for wireless microsensor networks. Proc. 33 ${ }^{\text {d }}$ Hawaii Intl. Conf. on System Sci. Maui, Hawaii, 8, 8020.

9. Hill J, Szewczyk R, Woo A, Hollar S, Culler D and Pister K (2000) System architecture directions for networked sensors. Proc. 9th Intl. Conf. on Architectural Support for Programming Languages \& Operating Sys. pp: 93-104.

10. Jone C, Sivalingam K, Agrawal P and Chen J (2001) A survey of energy efficient network protocols for wireless networks. J. Wireless Networks. 7, 343-358.

11. Madden S, Franklin MJ, Hellerstein JM and Hong W (2003) The design of an acquisitional query processor for sensor networks. Proc. ACM SIGMOD Intl. Conf. on Management of Data. pp: 491-502.

12. Yao Y and Gehrke JE (2002) The Cougar approach to in-network query processing in sensor networks. ACM Sigmod Record. 31 (3), pp: 9-18.
Research article

(C)Indian Society for Education and Environment (iSee)
"Crisis management using WSN" http://www.indjst.org
Gupta \& Dave Indian J.Sci.Technol. 